\title{
JORDAN DERIVATIONS ON PRIME RINGS AND THEIR APPLICATIONS IN BANACH ALGEBRAS, I
}

\author{
BYUNG-Do KIM
}

\begin{abstract}
The purpose of this paper is to prove that the noncommutative version of the Singer-Wermer Conjecture is affirmative under certain conditions. Let $A$ be a noncommutative Banach algebra. Suppose there exists a continuous linear Jordan derivation $D: A \rightarrow A$ such that $D(x)^{3}[D(x), x] \in \operatorname{rad}(A)$ for all $x \in A$. In this case, we show that $D(A) \subseteq \operatorname{rad}(A)$.
\end{abstract}

\section{Introduction}

Throughout, $R$ represents an associative ring and $A$ will be a complex Banach algebra. We write $[x, y]$ for the commutator $x y-y x$ for $x, y$ in a ring. Let $\operatorname{rad}(R)$ denote the (Jacobson) radical of a ring $R$. And a $\operatorname{ring} R$ is said to be (Jacobson) semisimple if its Jacobson $\operatorname{radical} \operatorname{rad}(R)$ is zero.

A ring $R$ is called $n$-torsion free if $n x=0$ implies $x=0$. Recall that $R$ is prime if $a R b=(0)$ implies that either $a=0$ or $b=0$, and is semiprime if $a R a=$ (0) implies $a=0$. On the other hand, let $X$ be an element of a normed algebra. Then for every $a \in X$ the spectral radius of $a$, denoted by $r(a)$, is defined by $r(a)=\inf \left\{\left\|a^{n}\right\|^{\frac{1}{n}}: n \in \mathbb{N}\right\}$. It is well-known that the following theorem holds: if $a$ is an element of a normed algebra, then $r(a)=\lim _{n \rightarrow \infty}\left\|a^{n}\right\|^{\frac{1}{n}}$ (see Bonsall and Duncan [1]).

An additive mapping $D$ from $R$ to $R$ is called a derivation if $D(x y)=$ $D(x) y+x D(y)$ holds for all $x, y \in R$. And an additive mapping $D$ from $R$ to $R$ is called a Jordan derivation if $D\left(x^{2}\right)=D(x) x+x D(x)$ holds for all $x \in R$.

Johnson and Sinclair [5] have proved that any linear derivation on a semisimple Banach algebra is continuous. A result of Singer and Wermer [12] states that every continuous linear derivation on a commutative Banach algebra maps the algebra into its radical. From these two results, we can conclude that there are no nonzero linear derivations on a commutative semisimple Banach algebra. Thomas [13] has proved that any linear derivation on a commutative Banach algebra maps the algebra into its radical.

Received September 3, 2012; Revised January 8, 2013.

2010 Mathematics Subject Classification. 16N60, 16W25, 17B40.

Key words and phrases. prime and semiprime ring, (Jacobson) radical, Jordan derivation. 
A noncommutative version of Singer and Wermer's Conjecture states that every continuous linear derivation on a noncommutative Banach algebra maps the algebra into its radical.

Vukman [15] has proved the following: Let $R$ be a 2-torsion free prime ring. If $D: R \longrightarrow R$ is a derivation such that $[D(x), x] D(x)=0$ for all $x \in R$, then $D=0$.

Moreover, using the above result, he has proved that the following holds: Let $A$ be a noncommutative semisimple Banach algebra. Suppose that $[D(x), x]$ $D(x)=0$ holds for all $x \in A$. In this case, $D=0$.

Kim [6] has showed that the following result holds: Let $R$ be a 3!-torsion free semiprime ring. Suppose there exists a Jordan derivation $D: R \rightarrow R$ such that

$$
[D(x), x] D(x)[D(x), x]=0
$$

for all $x \in R$. In this case, we have $[D(x), x]^{5}=0$ for all $x \in R$.

And, Kim [7] has showed that the following result holds: Let $A$ be a noncommutative Banach algebra. Suppose there exists a continuous linear Jordan derivation $D: A \rightarrow A$ such that $D(x)[D(x), x] D(x) \in \operatorname{rad}(A)$ for all $x \in A$. In this case, we have $D(A) \subseteq \operatorname{rad}(A)$.

In this paper, our aim is to prove the following results in the ring theory in order to apply it to the Banach algebra theory:

Let $R$ be a 7 !-torsion free prime ring. Suppose there exists a Jordan derivation $D: R \longrightarrow R$ such that

$$
D(x)^{3}[D(x), x]=0
$$

for all $x \in R$. In this case, we obtain $D(x)=0$ for all $x \in R$.

Let $A$ be a noncommutative Banach Algebra. Suppose there exists a continuous linear Jordan derivation $D: A \longrightarrow A$ such that

$$
D(x)^{3}[D(x), x] \in \operatorname{rad}(A)
$$

for all $x \in A$. In this case, we obtain $D(A) \subseteq \operatorname{rad}(A)$ for all $x \in A$.

\section{Preliminaries}

In this section, we review the basic results in prime and semiprime rings.

The following lemma is due to Chung and Luh [4].

Lemma 2.1. Let $R$ be a $n$ !-torsion free ring. Suppose there exist elements $y_{1}, y_{2}, \ldots, y_{n-1}, y_{n}$ in $R$ such that $\sum_{k=1}^{n} t^{k} y_{k}=0$ for all $t=1,2, \ldots, n$. Then we have $y_{k}=0$ for every positive integer $k$ with $1 \leq k \leq n$.

The following theorem is due to Brešar [3].

Theorem 2.2. Let $R$ be a 2-torsion free semiprime ring and let $D: R \longrightarrow R$ be a Jordan derivation. In this case, $D$ is a derivation.

The following theorem is due to Chung and Luh [4]. 
Theorem 2.3. Let $R$ be a semiprime ring with a derivation D. Suppose there exists a positive integer $n$ such that $(D x)^{n}=0$ for all $x \in R$ and suppose $R$ is $(n-1)$ !-torsion free. Then $D=0$.

\section{Main results}

We need the following notations. After this, by $S_{m}$ we denote the set $\{k \in$ $\mathbb{N} \mid 1 \leq k \leq m\}$ where $m$ is a positive integer. When $R$ is a ring, we shall denote the maps $B: R \times R \longrightarrow R, f, g: R \longrightarrow R$ by $B(x, y) \equiv[D(x), y]+$ $[D(y), x], f(x) \equiv[D(x), x], g(x) \equiv[f(x), x]$ for all $x, y \in R$, respectively. And we have the basic properties:

$$
\begin{aligned}
& B(x, y)=B(y, x), B(x, y z)=B(x, y) z+y B(x, z)+D(y)[z, x]+[y, x] D(z), \\
& B(x, x)=2 f(x), B\left(x, x^{2}\right)=2(f(x) x+x f(x)), x, y, z \in R .
\end{aligned}
$$

Lemma 3.1. Let $R$ be a 2-torsion free noncommutative prime ring. Suppose there exists a Jordan derivation $D: R \longrightarrow R$ such that

$$
[D(x), x]=0
$$

for all $x \in R$. Then we have $D(x)=0$ for all $x \in R$.

Proof. From Theorem 2.2, we see that $D$ is a derivation on $R$. Let

$$
f(x)=[D(x), x]=0, \quad x \in R .
$$

Substituting $x+y$ for $x$ in (1), we have

$$
f(x+y) \equiv f(x)+B(x, y)+[D(y), y]=0, x, y \in R .
$$

From (1) and (2), we obtain

$$
B(x, y)=0, \quad x, y \in R .
$$

Replacing $y x$ for $y$ in $(3)$, we have

$$
B(x, y) x+2 y f(x)+[y, x] D(x)=0, \quad x, y \in R .
$$

Combining (1), (3) with (4), we get

$$
[y, x] D(x)=0, x, y \in R .
$$

Substituting $z y$ for $y$ in (5), we have

$$
[z, x] y D(x)+z[y, x] D(x)=0, \quad x, y, z \in R .
$$

Combining (5) with (6), we obtain

$$
[z, x] y D(x)=0, \quad x, y, z \in R .
$$

Substituting $x+u$ for $x$ in (5),

(8) $[z, x] y D(x)+[z, u] y D(x)+[z, x] y D(u)+[z, u] y D(u)=0, u, x, y, z \in R$.

From (7) and (8),

$$
[z, u] y D(x)+[z, x] y D(u)=0, u, x, y, z \in R .
$$


Writing $y D(x) v[z, u] y$ for $y$ in (9), we have

$$
\begin{aligned}
& {[z, u] y D(x) v[z, u] y D(x)+[z, x] y D(x) v[z, u] y D(u) } \\
= & 0, u, v, x, y, z \in R .
\end{aligned}
$$

Combining (7) with (10), we get

$$
[z, u] y D(x) v[z, u] y D(x)=0, \quad u, v, x, y, z \in R .
$$

From (11) and the (semi)primeness of $R$,

$$
[z, u] y D(x)=0, \quad u, x, y, z \in R .
$$

By the primeness and noncommutativity of $R,(12)$ gives

$$
D(x)=0, \quad x \in R .
$$

Lemma 3.2. Let $R$ be a 2-torsion free noncommutative semiprime ring. Suppose there exists a Jordan derivation $D: R \longrightarrow R$ such that

$$
[[D(x), x], x]=0
$$

for all $x \in R$. Then we have $[D(x), x]=0$ for all $x \in R$.

Proof. From Theorem 2.2, we see that $D$ is a derivation on $R$.

Let

$$
g(x)=[[D(x), x], x]=0, \quad x \in R .
$$

Substituting $x+t y$ for $x$ in (13), we have

$$
\begin{aligned}
g(x+t y) \equiv & g(x)+t([f(x), y]+[B(x, y), x]) \\
& +t^{2} A(x, y)+t^{3} g(y)=0, x, y \in R, t \in S_{2},
\end{aligned}
$$

where $A(x, y)$ denotes the term satisfying the identity (14).

From (13) and (14), we obtain

(15) $\quad t([f(x), y]+[B(x, y), x])+t^{2} A(x, y)=0, x, y \in R, t \in S_{2}$.

Since $R$ is 2-torsion free, by Lemma 2.1, (15) yields

$$
[f(x), y]+[B(x, y), x]=0, \quad x, y \in R .
$$

Replacing $y x$ for $y$ in (16), we have

$$
\begin{aligned}
& {[f(x), y] x+y g(x)+[B(x, y), x] x} \\
& +3 y g(x)+3[y, x] f(x)+[[y, x], x] D(x)=0, \quad x, y \in R .
\end{aligned}
$$

Right multiplication of (16) by $x$ leads to

$$
[f(x), y] x+[B(x, y), x] x=0, \quad x, y \in R .
$$

Comparing (13), (17) and (18), we get

$$
3 y g(x)+3[y, x] f(x)+[[y, x], x] D(x)=0, \quad x, y \in R .
$$

From (13) and (19),

$$
3[y, x] f(x)+[[y, x], x] D(x)=0, \quad x, y \in R .
$$


From (20), we have

$$
3[y, x] g(x)+[[y, x], x] f(x)=0, \quad x, y \in R .
$$

From (13) with (21),

$$
[[y, x], x] f(x)=0, \quad x, y \in R .
$$

Writing $D(x) y$ for $y$ in (20), we have

$$
\begin{aligned}
& 3 D(x)[y, x] f(x)+3 f(x) y f(x)+D(x)[[y, x], x] D(x) \\
& +2 f(x)[y, x] D(x)+g(x) y D(x)=0, \quad x, y \in R .
\end{aligned}
$$

Left multiplication of $(20)$ by $D(x)$, we obtain

$$
3 D(x)[y, x] f(x)+D(x)[[y, x], x] D(x)=0, \quad x, y \in R .
$$

From (13), (23) and (24), we get

$$
3 f(x) y f(x)+2 f(x)[y, x] D(x)=0, \quad x, y \in R .
$$

Replacing $y D(x) w$ for $y$ in (25), we have

$$
\begin{aligned}
& 3 f(x) y D(x) w f(x)+2 f(x)[y, x] D(x) w D(x) \\
& +2 f(x) y f(x) w D(x)+2 f(x) y D(x)[w, x] D(x)=0, \quad w, x, y \in R .
\end{aligned}
$$

From (25) and (26), we get

$$
\begin{aligned}
& 3 f(x) y D(x) w f(x)-f(x) y f(x) w D(x) \\
& +2 f(x) y D(x)[w, x] D(x)=0, \quad w, x, y \in R .
\end{aligned}
$$

Substituting $w x$ for $w$ in (27), we have

$$
\begin{aligned}
& 3 f(x) y D(x) w x f(x)-f(x) y f(x) w x D(x) \\
& +2 f(x) y D(x)[w, x] x D(x)=0, \quad w, x, y \in R .
\end{aligned}
$$

Right multiplication of (27) by $x$ leads to

$$
\begin{aligned}
& 3 f(x) y D(x) w f(x) x-f(x) y f(x) w D(x) x \\
& +2 f(x) y D(x)[w, x] D(x) x=0, \quad w, x, y \in R .
\end{aligned}
$$

From (28) and (29), we get

$$
\begin{aligned}
& 3 f(x) y D(x) w g(x)-f(x) y f(x) w f(x) \\
& +2 f(x) y D(x)[w, x] f(x)=0, \quad w, x, y \in R .
\end{aligned}
$$

Comparing (13) and (30), we get

$$
f(x) y(f(x) w f(x)-2 y D(x)[w, x] f(x))=0, \quad w, x, y \in R .
$$

From (31), we obtain

$$
\begin{aligned}
& (f(x) w f(x)-2 D(x)[w, x] f(x)) y(f(x) w f(x) \\
& -2 D(x)[w, x] f(x)=0, \quad w, x, y \in R .
\end{aligned}
$$

Since $R$ is semiprime, (32) yields

$$
f(x) y f(x)-2 D(x)[y, x] f(x)=0, \quad x, y \in R .
$$


Putting $x y$ instead of $y$ in (16), we have

$$
x([f(x), y]+[B(x, y), x])+3 g(x) y+3 f(x)[y, x]+D(x)[[y, x], x]
$$

(34) $=0, \quad x, y \in R$.

Comparing (13), (16) and (34), we get

$$
3 f(x)[y, x]+D(x)[[y, x], x]=0, \quad x, y \in R .
$$

Writing $y D(x)$ for $y$ in $(35)$, we have

$$
\begin{aligned}
& 3 f(x)[y, x] D(x)+3 f(x) y f(x)+D(x)[[y, x], x] D(x) \\
& +2 D(x)[y, x] f(x)+D(x) y g(x)=0, \quad x, y \in R .
\end{aligned}
$$

From (35) and (36),

(37) $3 f(x) y f(x)+2 D(x)[y, x] f(x)+D(x) y g(x)=0, \quad x, y \in R$.

From (13) and (37), we have

$$
3 f(x) y f(x)+2 D(x)[y, x] f(x)=0, \quad x, y \in R .
$$

Combining (33) with (38),

$$
4 f(x) y f(x)=0, \quad x, y \in R .
$$

Since $R$ is 2-torsionfree, (39) gives

$$
f(x) y f(x)=0, \quad x, y \in R .
$$

Since $R$ is semiprime, (40) yields

$$
f(x)=[D(x), x]=0, \quad x \in R .
$$

Lemma 3.3. Let $R$ be a 7!-torsion free noncommutative prime ring. Suppose there exists a Jordan derivation $D: R \longrightarrow R$ such that

$$
D(x)^{5} y[[D(x), x], x]=0
$$

for all $x, y \in R$. Then we have $D(x)=0$ for all $x \in R$.

Proof. From Theorem 2.2, we see that $D$ is a derivation on $R$. Let

$$
D(x)^{5} y[[D(x), x], x]=0, \quad x, y \in R .
$$

Substituting $x+t z$ for $x$ in (41), we have

$$
\begin{aligned}
& D(x+t z)^{5} y g(x+t z) \\
= & D(x+t z)^{5} y[[D(x+t z), x+t z], x+t z] \\
\equiv & D(x)^{5} y g(x)+t\left\{\left(D(z) D(x)^{4}+D(x) D(z) D(x)^{3}\right.\right. \\
& \left.+D(x)^{2} D(z) D(x)^{2}+D(x)^{3} D(z) D(x)+D(x)^{4} D(z)\right) y g(x) \\
& \left.+D(x)^{5} y([[D(z), x], x]+[[D(x), z], x]+[f(x), z])\right\} \\
& +t^{2} C_{1}(x, y, z)+t^{3} C_{2}(x, y, z)+t^{4} C_{3}(x, y, z)+t^{5} C_{4}(x, y, z) \\
& +t^{6} C_{5}(x, y, z)+t^{7} C_{6}(x, y, z)+t^{8} D(z)^{5} y g(z) \\
= & 0, x, y, z \in R, t \in S_{7},
\end{aligned}
$$


where $C_{i}(x, y, z)(1 \leq i \leq 6)$ denotes the term satisfying the identity (42).

From (41) and (42), we obtain

$$
\begin{aligned}
& t\left\{\left(D(z) D(x)^{4}+D(x) D(z) D(x)^{3}\right.\right. \\
& \left.+D(x)^{2} D(z) D(x)^{2}+D(x)^{3} D(z) D(x)+D(x)^{4} D(z)\right) y g(x) \\
& \left.+D(x)^{5} y([[D(z), x], x]+[[D(x), z], x]+[f(x), z])\right\} \\
& +t^{2} C_{1}(x, y, z)+t^{3} C_{2}(x, y, z)+t^{4} C_{3}(x, y, z)+t^{5} C_{4}(x, y, z) \\
& +t^{6} C_{5}(x, y, z)+t^{7} C_{6}(x, y, z)=0, x, y, z \in R, t \in S_{7} .
\end{aligned}
$$

Since $R$ is 7 !-torsion free, by Lemma 2.1 , (43) yields

$$
\begin{aligned}
& \left\{D(z) D(x)^{4}+D(x) D(z) D(x)^{3}+D(x)^{2} D(z) D(x)^{2}\right. \\
& \left.+D(x)^{3} D(z) D(x)+D(x)^{4} D(z)\right\} y g(x) \\
& +D(x)^{5} y\{[[D(z), x], x]+[[D(x), z], x]+[f(x), z]\}=0, x, y, z \in R .
\end{aligned}
$$

Replacing $y g(x) u$ for $y$ in (44),

$$
\begin{aligned}
& \left(D(z) D(x)^{4}+D(x) D(z) D(x)^{3}+D(x)^{2} D(z) D(x)^{2}\right. \\
& \left.+D(x)^{3} D(z) D(x)+D(x)^{4} D(z)\right) y g(x) u g(x)
\end{aligned}
$$

$(45)+D(x)^{5} y g(x) u([[D(z), x], x]+[[D(x), z], x]+[f(x), z])=0, u, x, y, z \in R$.

Combining (41) with (45), we get

$$
\begin{aligned}
& \left(D(z) D(x)^{4}+D(x) D(z) D(x)^{3}+D(x)^{2} D(z) D(x)^{2}\right. \\
& \left.+D(x)^{3} D(z) D(x)+D(x)^{4} D(z)\right) y g(x) u g(x)=0, u, x, y, z \in R .
\end{aligned}
$$

Putting $u\left(D(z) D(x)^{4}+D(x) D(z) D(x)^{3}+D(x)^{2} D(z) D(x)^{2}+D(x)^{3} D(z) D(x)+\right.$ $\left.D(x)^{4} D(z)\right) y$ instead of $u$ in (46), we obtain

$$
\begin{aligned}
& \left(D(z) D(x)^{4}+D(x) D(z) D(x)^{3}+D(x)^{2} D(z) D(x)^{2}\right. \\
& \left.+D(x)^{3} D(z) D(x)+D(x)^{4} D(z)\right) y g(x) u\left(D(z) D(x)^{4}\right. \\
& +D(x) D(z) D(x)^{3}+D(x)^{2} D(z) D(x)^{2}+D(x)^{3} D(z) D(x) \\
& \left.+D(x)^{4} D(z)\right) y g(x)=0, u, x, y, z \in R .
\end{aligned}
$$

Since $R$ is semiprime, (47) yields

$$
\begin{aligned}
& \left(D(z) D(x)^{4}+D(x) D(z) D(x)^{3}+D(x)^{2} D(z) D(x)^{2}\right. \\
& \left.+D(x)^{3} D(z) D(x)+D(x)^{4} D(z)\right) y g(x)=0, x, y, z \in R .
\end{aligned}
$$

By using the same process of relations so obtained from (41) to (48) under the 5!-torsionfreeness repeatedly, we arrive at

$$
\begin{aligned}
& (D(z) D(v) D(w) D(p) D(q)+D(v) D(z) D(w) D(p) D(q)+\cdots \\
& +D(q) D(p) D(w) D(v) D(z)) y g(x)=0, u, v, w, p, q, x, y, z \in R .
\end{aligned}
$$

Let $u=v=w=p=q=z$ in (49).

$$
120 D(z)^{5} y g(x)=0, x, y, z \in R \text {. }
$$


Since $R$ is 7 !-torsionfree, (50) gives

$$
D(z)^{5} y g(x)=0, x, y, z \in R .
$$

Since $R$ is prime, it follows from (51) that

$$
D(z)^{5}=0, z \in R
$$

or

$$
g(x)=0, x \in R .
$$

Thus if (52) holds, then by Theorem 2.3,

$$
D(x)=0, x \in R .
$$

Thus if (53) holds, then by Lemma 3.2,

$$
[D(x), x]=0, x \in R .
$$

Hence by Lemma 3.1, (54) gives

$$
D(x)=0, x \in R .
$$

Therefore in any case, we have $D \equiv 0$.

Theorem 3.4. Let $R$ be a 7!-torsionfree noncommutative prime ring. Suppose there exists a Jordan derivation $D: R \longrightarrow R$ such that

$$
D(x)^{3}[D(x), x]=0
$$

for all $x \in R$. Then we have $D(x)=0$ for all $x \in R$.

Proof. By Theorem 2.2, we can see that $D$ is a derivation on $R$. Suppose

$$
D(x)^{3} f(x)=0, x \in R .
$$

Replacing $x+t y$ for $x$ in (55), we have

$$
\begin{aligned}
& D(x+t y)^{3}[D(x+t y), x+t y] \\
\equiv & D(x)^{3} f(x)+t\left\{D(y) D(x)^{2} f(x)+D(x) D(y) D(x) f(x)\right. \\
& \left.+D(x)^{2} D(y) f(x)+D(x)^{3} B(x, y)\right\}+t^{2} E_{1}(x, y)+t^{3} E_{2}(x, y) \\
& +t^{4} E_{3}(x, y)+t^{5} D(y)^{3} f(y)=0, x, y \in R, t \in S_{3},
\end{aligned}
$$

where $E_{i}(x, y), 1 \leq i \leq 3$, denotes the term satisfying the identity (56). From (55) and (56),

$$
\begin{aligned}
& t\left\{D(y) D(x)^{2} f(x)+D(x) D(y) D(x) f(x)+D(x)^{2} D(y) f(x)\right. \\
& \left.+D(x)^{3} B(x, y)\right\}+t^{2} E_{1}(x, y)+t^{3} E_{2}(x, y)+t^{4} E_{3}(x, y) \\
& =0, x, y \in R, t \in S_{4} .
\end{aligned}
$$

Since $R$ is 3!-torsionfree, by Lemma 2.1, (57) yields

$$
\begin{aligned}
& D(y) D(x)^{2} f(x)+D(x) D(y) D(x) f(x)+D(x)^{2} D(y) f(x) \\
& +D(x)^{3} B(x, y)=0, \quad x, y \in R .
\end{aligned}
$$


Let $y=x^{2}$ in (58). Then using (55), we get

$$
\begin{aligned}
& (D(x) x+x D(x)) D(x)^{2} f(x)+D(x)(D(x) x+x D(x)) D(x) f(x) \\
& +D(x)^{2}(D(x) x+x D(x)) f(x)+2 D(x)^{3}(f(x) x+x f(x)) \\
= & f(x) D(x)^{2} f(x)+(f(x) D(x)+D(x) f(x)) D(x) f(x) \\
& +f(x) D(x)^{2} f(x)+\left(f(x) D(x)^{2}+D(x) f(x) D(x)+D(x)^{2} f(x)\right) f(x) \\
& +(f(x) D(x)+D(x) f(x)) D(x) f(x)+2\left(f(x) D(x)^{2}+D(x) f(x) D(x)\right. \\
& \left.+D(x)^{2} f(x)\right) f(x) \\
= & 7 f(x) D(x)^{2} f(x)+5 D(x) f(x) D(x) f(x)+3 D(x)^{2} f(x)^{2}=0, \quad x \in R .
\end{aligned}
$$

Left multiplication of (59) by $D(x)^{2}$ leads to

(60) $\quad 7\left(D(x)^{2} f(x)\right)^{2}+5 D(x)^{3} f(x) D(x) f(x)+3 D(x)^{5} f(x)^{2}=0, \quad x \in R$.

Comparing (55) with (60),

$$
7\left(D(x)^{2} f(x)\right)^{2}=0, \quad x \in R .
$$

Since $R$ is 7 !-torsionfree, the above relation gives

$$
\left(D(x)^{2} f(x)\right)^{2}=0, \quad x \in R .
$$

On the other hand, we obtain from (55)

$$
\begin{aligned}
0= & {\left[D(x)^{3} f(x), x\right] } \\
= & f(x) D(x)^{2} f(x)+D(x) f(x) D(x) f(x)+D(x)^{2} f(x)^{2} \\
& +D(x)^{3} g(x), \quad x \in R .
\end{aligned}
$$

Left multiplication of $(62)$ by $D(x)^{2}$ leads to

$$
\begin{aligned}
& \left(D(x)^{2} f(x)\right)^{2}+D(x)^{3} f(x) D(x) f(x)+D(x)^{4} f(x)^{2} \\
& +D(x)^{5} g(x)=0, \quad x \in R .
\end{aligned}
$$

Comparing (55), (61) and (63),

$$
D(x)^{5} g(x)=0, \quad x \in R .
$$

From (59) and (62), we get

$$
4 f(x) D(x)^{2} f(x)+2 D(x) f(x) D(x) f(x)-3 D(x)^{3} g(x)=0, \quad x \in R .
$$

Combining (59) with (65),

$$
3\left(2 f(x) D(x)^{2} f(x)-2 D(x)^{2} f(x)^{2}-5 D(x)^{3} g(x)\right)=0, \quad x \in R .
$$

Since $R$ is 3 !-torsion-free, the above relation gives

$$
2 f(x) D(x)^{2} f(x)-2 D(x)^{2} f(x)^{2}-5 D(x)^{3} g(x)=0, \quad x \in R .
$$

Writing $x y$ for $y$ in (58), we have

$$
\begin{aligned}
& x D(y) D(x)^{2} f(x)+D(x) y D(x)^{2} f(x)+D(x) x D(y) D(x) f(x) \\
& +D(x)^{2} y D(x) f(x)+D(x)^{2} x D(y) f(x)+D(x)^{3} y f(x) \\
& +D(x)^{3}(2 f(x) y+x B(x, y)+D(x)[y, x])=0, \quad x, y \in R .
\end{aligned}
$$


Left multiplication of (58) by $x$ leads to

$$
\begin{aligned}
& x D(y) D(x)^{2} f(x)+x D(x) D(y) D(x) f(x)+x D(x)^{2} D(y) f(x) \\
& +x D(x)^{3} B(x, y)=0, \quad x, y \in R .
\end{aligned}
$$

From (67) and (68), we arrive at

$$
\begin{aligned}
& D(x) y D(x)^{2} f(x)+f(x) D(y) D(x) f(x)+D(x)^{2} y D(x) f(x) \\
& +f(x) D(x) D(y) f(x)+D(x) f(x) D(y) f(x)+D(x)^{3} y f(x) \\
& +2 D(x)^{3} f(x) y+D(x)^{3} x B(x, y)-x D(x)^{3} B(x, y) \\
& +D(x)^{4}[y, x]=0, \quad x, y \in R .
\end{aligned}
$$

By (55) and (69), it is obvious that

$$
\begin{aligned}
& D(x) y D(x)^{2} f(x)+f(x) D(y) D(x) f(x)+D(x)^{2} y D(x) f(x) \\
& +f(x) D(x) D(y) f(x)+D(x) f(x) D(y) f(x)+D(x)^{3} y f(x) \\
& +f(x) D(x)^{2} B(x, y)+D(x) f(x) D(x) B(x, y)+D(x)^{2} f(x) B(x, y) \\
& +D(x)^{4}[y, x]=0, \quad x, y \in R .
\end{aligned}
$$

Left multiplication of (70) by $D(x)^{3}$ gives

$$
\begin{aligned}
& D(x)^{4} y D(x)^{2} f(x)+D(x)^{3} f(x) D(y) D(x) f(x)+D(x)^{5} y D(x) f(x) \\
& +D(x)^{3} f(x) D(x) D(y) f(x)+D(x)^{4} f(x) D(y) f(x)+D(x)^{6} y f(x) \\
& +D(x)^{3} f(x) D(x)^{2} B(x, y)+D(x)^{4} f(x) D(x) B(x, y) \\
& +D(x)^{5} f(x) B(x, y)+D(x)^{7}[y, x]=0, \quad x, y \in R .
\end{aligned}
$$

Combining (55) with (71),

$$
\begin{aligned}
& D(x)^{4} y D(x)^{2} f(x)+D(x)^{5} y D(x) f(x)+D(x)^{6} y f(x) \\
& +D(x)^{7}[y, x]=0, \quad x, y \in R .
\end{aligned}
$$

Replacing $y x$ for $y$ in (72),

$$
\begin{aligned}
& D(x)^{4} y x D(x)^{2} f(x)+D(x)^{5} y x D(x) f(x)+D(x)^{6} y x f(x) \\
& +D(x)^{7}[y, x] x=0, \quad x, y \in R .
\end{aligned}
$$

Right multiplication of (72) by $x$ leads to

$$
\begin{aligned}
& D(x)^{4} y D(x)^{2} f(x) x+D(x)^{5} y D(x) f(x) x+D(x)^{6} y f(x) x \\
& +D(x)^{7}[y, x] x=0, \quad x, y \in R .
\end{aligned}
$$

Combining (73) with (74),

$$
\begin{aligned}
& D(x)^{4} y\left(f(x) D(x) f(x)+D(x) f(x)^{2}+D(x)^{2} g(x)\right) \\
& +D(x)^{5} y\left(f(x)^{2}+D(x) g(x)\right)+D(x)^{6} y g(x)=0, \quad x, y \in R .
\end{aligned}
$$

Writing $y D(x)^{4}$ for $y$ in (75), we get

$$
D(x)^{4} y\left(D(x)^{4} f(x) D(x) f(x)+D(x)^{5} f(x)^{2}+D(x)^{6} g(x)\right)
$$

$(76)+D(x)^{5} y\left(D(x)^{4} f(x)^{2}+D(x)^{5} g(x)\right)+D(x)^{6} y D(x)^{4} g(x)=0, \quad x, y \in R$. 
From (55), (64) and (76),

$$
D(x)^{6} y D(x)^{4} g(x)=0, \quad x, y \in R .
$$

Comparing (55), (75) and (77),

$$
\begin{aligned}
& D(x)^{4} y D(x)^{4} g(x) z\left(D(x)^{2} f(x) D(x) f(x)+D(x)^{4} g(x)\right) \\
& +D(x)^{5} y D(x)^{4} g(x) z\left(D(x)^{2} f(x)^{2}+D(x)^{3} g(x)\right)=0, \quad x, y, z \in R .
\end{aligned}
$$

Left multiplication of (65) by $D(x)$ leads to

(79) $4 D(x) f(x) D(x)^{2} f(x)+2 D(x)^{2} f(x) D(x) f(x)-3 D(x)^{4} g(x)=0, \quad x \in R$.

Left multiplication of (66) by $D(x)$ yields

$$
2 D(x) f(x) D(x)^{2} f(x)-2 D(x)^{3} f(x)^{2}-5 D(x)^{4} g(x)=0, \quad x \in R .
$$

From (55) and (80),

$$
2 D(x) f(x) D(x)^{2} f(x)-5 D(x)^{4} g(x)=0, \quad x \in R .
$$

From (79) and (81), we have

$$
2 D(x)^{2} f(x) D(x) f(x)+7 D(x)^{4} g(x)=0, \quad x \in R .
$$

From (78) and (82), we arrive at

$$
\begin{aligned}
& D(x)^{4} y D(x)^{4} g(x) z\left(2 D(x)^{2} f(x) D(x) f(x)+2 D(x)^{4} g(x)\right) \\
& +2 D(x)^{5} y D(x)^{4} g(x) z\left(D(x)^{2} f(x)^{2}+D(x)^{3} g(x)\right) \\
= & D(x)^{4} y D(x)^{4} g(x) z\left(-7 D(x)^{4} g(x)+2 D(x)^{4} g(x)\right) \\
& +2 D(x)^{5} y D(x)^{4} g(x) z\left(D(x)^{2} f(x)^{2}+D(x)^{3} g(x)\right) \\
= & -5 D(x)^{4} y D(x)^{4} g(x) z D(x)^{4} g(x) \\
& +2 D(x)^{5} y D(x)^{4} g(x) z\left(D(x)^{2} f(x)^{2}+D(x)^{3} g(x)\right) \\
= & 0, \quad x, y, z \in R .
\end{aligned}
$$

Substituting $g(x) y$ for $y$ in (83), it follows that

$$
\begin{aligned}
& -5 D(x)^{4} g(x) y D(x)^{4} g(x) z D(x)^{4} g(x) \\
& +2 D(x)^{5} g(x) y D(x)^{4} g(x) z\left(D(x)^{2} f(x)^{2}+D(x)^{3} g(x)\right) \\
= & 0, \quad x, y, z \in R .
\end{aligned}
$$

Comparing (64) and (84),

$$
-5 D(x)^{4} g(x) y D(x)^{4} g(x) z D(x)^{4} g(x)=0, \quad x, y, z \in R .
$$

Since $R$ is 5 !-torsion-free, the above relation yields

$$
D(x)^{4} g(x) y D(x)^{4} g(x) z D(x)^{4} g(x)=0, \quad x, y, z \in R .
$$

Thus by the semiprimeness of $R,(85)$ gives

$$
D(x)^{4} g(x)=0, \quad x \in R .
$$

From (81) and (86),

$$
2 D(x) f(x) D(x)^{2} f(x)=0, \quad x \in R .
$$


Since $R$ is 5 !-torsion-free, the above relation gives

$$
D(x) f(x) D(x)^{2} f(x)=0, \quad x \in R .
$$

From (82) and (86), we have

$$
2 D(x)^{2} f(x) D(x) f(x)=0, \quad x \in R .
$$

Since $R$ is 2 !-torsion-free, the above relation gives

$$
D(x)^{2} f(x) D(x) f(x)=0, \quad x \in R .
$$

Substituting $y D(x)^{2}$ for $y$ in (75), it follows that

$$
D(x)^{4} y\left(D(x)^{2} f(x) D(x) f(x)+D(x)^{3} f(x)^{2}+D(x)^{4} g(x)\right)
$$

$(89)+D(x)^{5} y\left(D(x)^{2} f(x)^{2}+D(x)^{3} g(x)\right)+D(x)^{6} y D(x)^{2} g(x)=0, \quad x, y \in R$.

From (55), (86), (88) and (89),

(90) $D(x)^{5} y\left(D(x)^{2} f(x)^{2}+D(x)^{3} g(x)\right)+D(x)^{6} y D(x)^{2} g(x)=0, \quad x, y \in R$.

Writing $y D(x)$ for $y$ in $(90)$, we get

(91) $D(x)^{5} y\left(D(x)^{3} f(x)^{2}+D(x)^{4} g(x)\right)+D(x)^{6} y D(x)^{3} g(x)=0, \quad x, y \in R$.

Combining (55), (86) with (91),

$$
D(x)^{6} y D(x)^{3} g(x)=0, \quad x, y \in R .
$$

Replacing $y D(x) f(x)$ for $y$ in (72), it follows that

$$
\begin{aligned}
& D(x)^{4} y D(x) f(x) D(x)^{2} f(x)+D(x)^{5} y D(x) f(x) D(x) f(x) \\
& +D(x)^{6} y D(x) f(x)^{2}+D(x)^{7}[y, x] D(x) f(x) \\
& +D(x)^{7} y\left(f(x)^{2}+D(x) g(x)\right)=0, \quad x, y \in R .
\end{aligned}
$$

From (87) and (93),

$$
D(x)^{5} y D(x) f(x) D(x) f(x)+D(x)^{6} y D(x) f(x)^{2}
$$$$
\text { Right multiplication of }(72) \text { by } D(x) f(x) \text { leads to }
$$

$$
\begin{aligned}
& D(x)^{4} y D(x)^{2} f(x) D(x) f(x)+D(x)^{5} y D(x) f(x) D(x) f(x) \\
& +D(x)^{6} y f(x) D(x) f(x)+D(x)^{7}[y, x] D(x) f(x)=0, \quad x, y \in R .
\end{aligned}
$$

Combining (88) with (95),

$$
\begin{aligned}
& D(x)^{5} y D(x) f(x) D(x) f(x)+D(x)^{6} y f(x) D(x) f(x) \\
& +D(x)^{7}[y, x] D(x) f(x)=0, \quad x, y \in R .
\end{aligned}
$$

Combining (94) with (96),

$$
\begin{aligned}
& D(x)^{6} y\left(D(x) f(x)^{2}-f(x) D(x) f(x)\right)+D(x)^{7} y\left(f(x)^{2}+D(x) g(x)\right) \\
= & 0, \quad x, y \in R .
\end{aligned}
$$


Writing $y D(x)^{2}$ for $y$ in (97), we get

$$
\begin{aligned}
& D(x)^{6} y\left(D(x)^{3} f^{2}-D(x)^{2} f(x) D(x) f(x)\right) \\
& +D(x)^{7} y\left(D(x)^{2} f(x)^{2}+D(x)^{3} g(x)\right)=0, \quad x, y \in R .
\end{aligned}
$$

From (55), (88), (92) and (98),

$$
D(x)^{7} y D(x)^{2} f(x)^{2}=0, \quad x, y \in R .
$$

Writing $y D(x)^{2} f(x)^{2} z D(x)$ for $y$ in (97),

$$
\begin{aligned}
& D(x)^{6} y D(x)^{2} f(x)^{2} z\left(D(x)^{2} f(x)^{2}-D(x) f(x) D(x) f(x)\right) \\
& +D(x)^{7} y D(x)^{2} f(x)^{2} z\left(D(x) f(x)^{2}+D(x)^{2} g(x)\right)=0, \quad x, y, z \in R .
\end{aligned}
$$

From (99) and (100), we obtain

(101) $D(x)^{6} y D(x)^{2} f(x)^{2} z\left(D(x)^{2} f(x)^{2}-D(x) f(x) D(x) f(x)\right)=0, x, y, z \in R$.

From (59) and (62),

$$
\begin{aligned}
& 7\left(-D(x) f(x) D(x) f(x)-D(x)^{2} f(x)^{2}-D(x)^{3} g(x)\right) \\
& +5 D(x) f(x) D(x) f(x)+3 D(x)^{2} f(x)^{2} \\
(102)= & -2 D(x) f(x) D(x) f(x)+4 D(x)^{2} f(x)^{2}-7 D(x)^{3} g(x)=0, \quad x \in R .
\end{aligned}
$$

From (92) and (102),

$D(x)^{6} y D(x)^{2} f(x)^{2} z\left(-2 D(x) f(x) D(x) f(x)+4 D(x)^{2} f(x)^{2}\right)=0, \quad x, y, z \in R$.

Since $R$ is 2 !-torsion-free, the above relation gives

$$
D(x)^{6} y D(x)^{2} f(x)^{2} z\left(D(x) f(x) D(x) f(x)-2 D(x)^{2} f(x)^{2}\right)=0, \quad x, y, z \in R .
$$

From (101) and (103), we get

$$
D(x)^{6} y D(x)^{2} f(x)^{2} z D(x)^{2} f(x)^{2}=0, x, y, z \in R .
$$

The above relation yields

$$
D(x)^{6} y D(x)^{2} f(x)^{2} z D(x)^{6} y D(x)^{2} f(x)^{2}=0, x, y, z \in R .
$$

Thus by the primeness of $R$, (104) gives

$$
D(x)^{6} y D(x)^{2} f(x)^{2}=0, \quad x, y \in R .
$$

Writing $D(x) y D(x)^{2} f(x)^{2} z D(x)$ for $y$ in (75),

$$
\begin{aligned}
& D(x)^{5} y D(x)^{2} f(x)^{2} z\left(D(x) f(x) D(x) f(x)+D(x)^{2} f(x)^{2}+D(x)^{3} g(x)\right) \\
& +D(x)^{6} y D(x)^{2} f(x)^{2} z\left(D(x) f(x)^{2}+D(x)^{2} g(x)\right) \\
(106) \quad & +D(x)^{7} y D(x)^{2} f(x)^{2} z D(x) g(x)=0, \quad x, y, z \in R .
\end{aligned}
$$

Combining (105) with (106),

$$
D(x)^{5} y D(x)^{2} f(x)^{2} z\left(D(x) f(x) D(x) f(x)+D(x)^{2} f(x)^{2}+D(x)^{3} g(x)\right)
$$

$(107)=0, \quad x, y, z \in R$. 
From (62) and (107), we have

$$
D(x)^{5} y D(x)^{2} f(x)^{2} z f(x) D(x)^{2} f(x)=0, \quad x, y, z \in R .
$$

Right multiplication of (66) by $D(x)^{5} y D(x)^{2} f(x)^{2} z$ leads to

$$
D(x)^{5} y D(x)^{2} f(x)^{2} z\left(2 f(x) D(x)^{2} f(x)-2 D(x)^{2} f(x)^{2}-5 D(x)^{3} g(x)\right)
$$

$(109)=0, \quad x, y, z \in R$.

From (108) and (109),

$$
D(x)^{5} y D(x)^{2} f(x)^{2} z\left(2 D(x)^{2} f(x)^{2}+5 D(x)^{3} g(x)\right)=0, \quad x, y, z \in R .
$$

Writing $y D(x)^{2} f(x)^{2} z$ for $y$ in (90), we get

$$
\begin{aligned}
& D(x)^{5} y D(x)^{2} f(x)^{2} z\left(D(x)^{2} f(x)^{2}+D(x)^{3} g(x)\right) \\
& \left.+D(x)^{6} y D(x)^{2} f(x)^{2} z D(x)^{2} g(x)\right)=0, \quad x, y, z \in R .
\end{aligned}
$$

Combining (105) with (111),

$$
D(x)^{5} y D(x)^{2} f(x)^{2} z\left(D(x)^{2} f(x)^{2}+D(x)^{3} g(x)\right)=0, \quad x, y, z \in R .
$$

Comparing (110) and (112),

$$
3 D(x)^{5} y D(x)^{2} f(x)^{2} z D(x)^{3} g(x)=0, \quad x, y, z \in R .
$$

Since $R$ is 3 !-torsion-free, the above relation gives

$$
D(x)^{5} y D(x)^{2} f(x)^{2} z D(x)^{3} g(x)=0, x, y, z \in R .
$$

From (112) and (113), we obtain

$$
D(x)^{5} y D(x)^{2} f(x)^{2} z D(x)^{2} f(x)^{2}=0, x, y, z \in R .
$$

Thus by the primeness of $R$, (114) gives

$$
D(x)^{5} y D(x)^{2} f(x)^{2}=0, \quad x, y \in R .
$$

From (90) and (115),

$$
D(x)^{5} y D(x)^{3} g(x)+D(x)^{6} y D(x)^{2} g(x)=0, x, y \in R .
$$

Writing $y D(x)^{3} g(x) z$ for $y$ in (116),

$$
D(x)^{5} y D(x)^{3} g(x) z D(x)^{3} g(x)+D(x)^{6} y D(x)^{3} g(x) z D(x)^{2} g(x)
$$

From (92) and (117), we have

$$
D(x)^{5} y D(x)^{3} g(x) z D(x)^{3} g(x)=0, \quad x, y, z \in R .
$$

From (118),

$$
D(x)^{5} y D(x)^{3} g(x) z D(x)^{5} y D(x)^{3} g(x)=0, \quad x, y, z \in R .
$$

Thus by the primeness of $R,(114)$ gives

$$
D(x)^{5} y D(x)^{3} g(x)=0, \quad x, y \in R .
$$


From (116) and (120), we get

$$
D(x)^{6} y D(x)^{2} g(x)=0, \quad x, y \in R .
$$

Right multiplication of (72) by $z D(x)^{2} f(x)^{2}$ leads to

$$
D(x)^{4} y D(x)^{2} f(x) z D(x)^{2} f(x)^{2}+D(x)^{5} y D(x) f(x) z D(x)^{2} f(x)^{2}
$$

$(122)+D(x)^{6} y f(x) z D(x)^{2} f(x)^{2}+D(x)^{7}[y, x] z D(x)^{2} f(x)^{2}=0, \quad x, y, z \in R$.

Combining (115) with (122), we arrive at

$$
D(x)^{4} y D(x)^{2} f(x) z D(x)^{2} f(x)^{2}=0, \quad x, y, z \in R .
$$

Writing $f z$ for $z$ in (123), we get

$$
D(x)^{4} y D(x)^{2} f(x)^{2} z D(x)^{2} f(x)^{2}=0, \quad x, y, z \in R .
$$

From (124), we have

$$
D(x)^{4} y D(x)^{2} f(x)^{2} z D(x)^{4} y D(x)^{2} f(x)^{2}=0, \quad x, y, z \in R .
$$

Thus by the primeness of $R,(125)$ gives

$$
D(x)^{4} y D(x)^{2} f(x)^{2}=0, \quad x, y \in R .
$$

Right multiplication of (72) by $D(x) f(x)$ leads to

$$
\begin{aligned}
& D(x)^{4} y D(x)^{2} f(x) D(x) f(x)+D(x)^{5} y D(x) f(x) D(x) f(x) \\
& +D(x)^{6} y f(x) D(x) f(x)+D(x)^{7}[y, x] D(x) f(x)=0, \quad x, y \in R .
\end{aligned}
$$

Comparing (88) and (127),

$$
-D(x)^{5} y D(x) f(x) D(x) f(x)+D(x)^{6} y\left(D(x) f(x)^{2}-f(x) D(x) f(x)\right)
$$

$(128)+D(x)^{7} y f(x)^{2}=0, \quad x, y \in R$.

Writing $y D(x)$ for $y$ in (128),

$$
\begin{aligned}
& -D(x)^{5} y D(x)^{2} f(x) D(x) f(x)+D(x)^{6} y\left(D(x)^{2} f(x)^{2}\right. \\
& -D(x) f(x) D(x) f(x))+D(x)^{7} y D(x) f(x)^{2}=0, \quad x, y \in R .
\end{aligned}
$$

Combining (88), (126) with (129), we have

$$
-D(x)^{6} y D(x) f(x) D(x) f(x)+D(x)^{7} y D(x) f(x)^{2}=0, \quad x, y \in R .
$$

From (130),

(131) $D(x)^{6} y(-2 D(x) f(x) D(x) f(x))+2 D(x)^{7} y D(x) f(x)^{2}=0, \quad x, y \in R$.

Comparing (102) and (131),

$(132)$

$$
D(x)^{6} y\left(4 D(x)^{2} f(x)^{2}+7 D(x)^{3} g(x)\right)+2 D(x)^{7} y D(x) f(x)^{2}=0, \quad x, y \in R .
$$

Combining (91), (105) with (132),

$$
2 D(x)^{7} y D(x) f(x)^{2}=0, \quad x, y \in R .
$$

Since $R$ is 3 !-torsion-free, the above relation gives

$$
D(x)^{7} y D(x) f(x)^{2}=0, \quad x, y \in R .
$$


Right multiplication of (72) by $f(x)$ gives

$$
D(x)^{5} y D(x) f(x)^{2}+D(x)^{6} y f(x)^{2}+D(x)^{7}[y, x] f(x)=0, \quad x, y \in R .
$$

Left multiplication of (134) by $D(x)$ leads to

$$
D(x)^{6} y D(x) f(x)^{2}+D(x)^{7} y f(x)^{2}+D(x)^{8}[y, x] f(x)=0, \quad x, y \in R .
$$

Right multiplication of (135) by $z D(x) f(x)^{2}$ yields

$$
D(x)^{6} y D(x) f(x)^{2} z D(x) f(x)^{2}+D(x)^{7} y f(x)^{2} z D(x) f(x)^{2}
$$

Combining (133) with (136),

$$
D(x)^{6} y D(x) f(x)^{2} z D(x) f(x)^{2}=0, \quad x, y, z \in R .
$$

It follows from (137) that

$$
D(x)^{6} y D(x) f(x)^{2} z D(x)^{6} y D(x) f(x)^{2}=0, \quad x, y, z \in R .
$$

By the primeness of $R$, we get from (138)

$$
D(x)^{6} y D(x) f(x)^{2}=0, \quad x, y \in R .
$$

Right multiplication of (72) by $z D(x) f(x)^{2}$ leads to

$$
D(x)^{4} y D(x)^{2} f(x) z D(x) f(x)^{2}+D(x)^{5} y D(x) f(x) z D(x) f(x)^{2}
$$

$(140)+D(x)^{6} y f(x) z D(x) f(x)^{2}+D(x)^{7}[y, x] z D(x) f(x)^{2}=0, \quad x, y, z \in R$.

Combining (133), (139) with (140),

$$
D(x)^{4} y D(x)^{2} f(x) z D(x) f(x)^{2}+D(x)^{5} y D(x) f(x) z D(x) f(x)^{2}
$$

$$
=0, \quad x, y, z \in R \text {. }
$$

Replacing $f(x) z$ for $z$ in (141), it follows that

$$
D(x)^{4} y D(x)^{2} f(x)^{2} z D(x) f(x)^{2}+D(x)^{5} y D(x) f(x)^{2} z D(x) f(x)^{2}
$$

(142) $=0, \quad x, y, z \in R$.

Comparing (126) and (142),

$$
D(x)^{5} y D(x) f(x)^{2} z D(x) f(x)^{2}=0, \quad x, y, z \in R .
$$

It follows from (143) that

$$
D(x)^{5} y D(x) f(x)^{2} z D(x)^{5} y D(x) f(x)^{2}=0, \quad x, y, z \in R .
$$

By the primeness of $R$, we obtain from (144)

$$
D(x)^{5} y D(x) f(x)^{2}=0, \quad x, y \in R .
$$

Combining (141) with (145),

$$
D(x)^{4} y D(x)^{2} f(x) z D(x) f(x)^{2}=0, \quad x, y, z \in R .
$$

Replacing $y D(x)$ for $y$ in $(72)$,

$$
\begin{aligned}
& D(x)^{5} y D(x)^{2} f(x)+D(x)^{6} y D(x) f(x)+D(x)^{7}[y, x] D(x) \\
& +D(x)^{7} y f(x)=0, \quad x, y \in R .
\end{aligned}
$$


Left multiplication of (72) by $D(x)$ leads to

$$
\begin{aligned}
& D(x)^{5} y D(x)^{2} f(x)+D(x)^{6} y D(x) f(x)+D(x)^{7} y f(x) \\
& +D(x)^{8}[y, x]=0, \quad x, y \in R .
\end{aligned}
$$

Combining (147) with (148), we have

$$
D(x)^{7}[y, x] D(x)-D(x)^{8}[y, x]=0, \quad x, y \in R .
$$

Replacing $y x$ for $y$ in (149),

$$
D(x)^{7}[y, x] x D(x)-D(x)^{8}[y, x] x=0, \quad x, y \in R .
$$

Right multiplication of (149) by $x$ leads to

$$
D(x)^{7}[y, x] D(x) x-D(x)^{8}[y, x] x=0, \quad x, y \in R .
$$

Combining (150) with (151), we get

$$
D(x)^{7}[y, x] f(x)=0, \quad x, y \in R .
$$

Right multiplication of (72) by $f(x)$ leads to

$$
\begin{aligned}
& D(x)^{4} y D(x)^{2} f(x)^{2}+D(x)^{5} y D(x) f(x)^{2}+D(x)^{6} y f(x)^{2} \\
& +D(x)^{7}[y, x] f(x)=0, \quad x, y \in R .
\end{aligned}
$$

From (126), (145), (152) and (153), we conclude that

$$
D(x)^{6} y f(x)^{2}=0, \quad x, y \in R .
$$

Left multiplication of (75) by $D(x)$ leads to

$$
\begin{aligned}
& D(x)^{5} y\left(f(x) D(x) f(x)+D(x) f(x)^{2}+D(x)^{2} g(x)\right) \\
& +D(x)^{6} y\left(f(x)^{2}+D(x) g(x)\right)+D(x)^{7} y g(x)=0, \quad x, y \in R .
\end{aligned}
$$

From (145), (154) and (155), we get

$$
\begin{aligned}
& D(x)^{5} y\left(f(x) D(x) f(x)+D(x)^{2} g(x)\right)+D(x)^{6} y D(x) g(x) \\
& +D(x)^{7} y g(x)=0, \quad x, y \in R .
\end{aligned}
$$

Replacing $y D(x)$ for $y$ in (149), it follows that

$$
D(x)^{7}[y, x] D(x)^{2}+D(x)^{7} y f(x) D(x)-D(x)^{8}[y, x] D(x)
$$

Right multiplication of (149) by $D(x)$ gives

$$
D(x)^{7}[y, x] D(x)^{2}-D(x)^{8}[y, x] D(x)=0, \quad x, y \in R .
$$

From (157) and (158), we have

$$
D(x)^{7} y f(x) D(x)-D(x)^{8} y f(x)=0, \quad x, y \in R .
$$

Right multiplication of (159) by $f(x)$ leads to

$$
D(x)^{7} y f(x) D(x) f(x)-D(x)^{8} y f(x)^{2}=0, \quad x, y \in R .
$$

From (154) and (160),

$$
D(x)^{7} y f(x) D(x) f(x)=0, \quad x, y \in R .
$$


Replacing $y f(x) D(x) f(x) z$ for $y$ in (75), it follows that

$$
\begin{aligned}
& D(x)^{4} y f(x) D(x) f(x) z\left(f(x) D(x) f(x)+D(x) f(x)^{2}+D(x)^{2} g(x)\right) \\
& +D(x)^{5} y f(x) D(x) f(x) z\left(f(x)^{2}+D(x) g(x)\right) \\
(162) \quad & +D(x)^{6} y f(x) D(x) f(x) z g(x)=0, \quad x, y, z \in R .
\end{aligned}
$$

Left multiplication of (162) by $D(x)^{2}$ gives

$$
\begin{aligned}
& D(X)^{6} y f(x) D(x) f(x) z\left(f(x) D(x) f(x)+D(x) f(x)^{2}+D(x)^{2} g(x)\right) \\
& +D(x)^{7} y f(x) D(x) f(x) z\left(f(x)^{2}+D(x) g(x)\right) \\
(163) \quad & +D(x)^{8} y f(x) D(x) f(x) z g(x)=0, \quad x, y, z \in R .
\end{aligned}
$$

From (161) and (163),

$$
\begin{aligned}
& D(x)^{6} y f(x) D(x) f(x) z\left(f(x) D(x) f(x)+D(x) f(x)^{2}+D(x)^{2} g(x)\right) \\
(164)= & 0, \quad x, y, z \in R .
\end{aligned}
$$

From (121), (145) and (164), we obtain

$$
D(x)^{6} y f(x) D(x) f(x) z f(x) D(x) f(x)=0, \quad x, y, z \in R .
$$

From (165),

(166) $\quad D(x)^{6} y f(x) D(x) f(x) z D(x)^{6} y f(x) D(x) f(x)=0, \quad x, y, z \in R$.

Since $R$ is prime, we get from (166)

$$
D(x)^{6} y f(x) D(x) f(x)=0, \quad x, y \in R .
$$

Right multiplication of (156) by $z f(x) D(x) f(x)$ leads to

$$
\begin{aligned}
& D(x)^{5} y\left(f(x) D(x) f(x)+D(x)^{2} g(x)\right) z f(x) D(x) f(x) \\
& +D(x)^{6} y D(x) g(x) z f(x) D(x) f(x)+D(x)^{7} y g(x) z f(x) D(x) f(x) \\
(168) \quad= & 0, \quad x, y, z \in R .
\end{aligned}
$$

From (167) and (168), we obtain

$$
\text { (169) }=0, \quad x, y, z \in R \text {. }
$$$$
D(x)^{5} y\left(f(x) D(x) f(x)+D(x) f(x)^{2}+D(x)^{2} g(x)\right) z f(x) D(x) f(x)
$$

On the other hand, right multiplication of (156) by $z D(x)^{2} g(x)$ leads to

$$
\begin{aligned}
& D(x)^{5} y\left(f(x) D(x) f(x)+D(x)^{2} g(x)\right) z D(x)^{2} g(x) \\
& +D(x)^{6} y D(x) g(x) z D(x)^{2} g(x)+D(x)^{7} y g(x) z D(x)^{2} g(x) \\
= & 0, \quad x, y, z \in R .
\end{aligned}
$$

From (121) and (170),

(171) $\quad D(x)^{5} y\left(f(x) D(x) f(x)+D(x)^{2} g(x)\right) z D(x)^{2} g(x)=0, \quad x, y, z \in R$.

From (169) and (171), we have

$$
\begin{aligned}
& D(x)^{5} y\left(f(x) D(x) f(x)+D(x)^{2} g(x)\right) z(f(x) D(x) f(x) \\
& \left.+D(x)^{2} g(x)\right)=0, \quad x, y, z \in R .
\end{aligned}
$$


From (172),

$$
\begin{aligned}
& D(x)^{5} y\left(f(x) D(x) f(x)+D(x)^{2} g(x)\right) z D(x)^{5} y(f(x) D(x) f(x) \\
& \left.+D(x)^{2} g(x)\right)=0, \quad x, y, z \in R .
\end{aligned}
$$

Since $R$ is prime, (173) gives

$$
D(x)^{5} y\left(f(x) D(x) f(x)+D(x)^{2} g(x)\right)=0, \quad x, y \in R .
$$

From (156) and (174), we get

$$
D(x)^{6} y D(x) g(x)+D(x)^{7} y g(x)=0, \quad x, y \in R .
$$

Replacing $y D(x)$ for $y$ in (175),

$$
D(x)^{6} y D(x)^{2} g(x)+D(x)^{7} y D(x) g(x)=0, \quad x, y \in R .
$$

From (121) and (176),

$$
D(x)^{7} y D(x) g(x)=0, \quad x, y \in R .
$$

Replacing $y D(x) g(x) z$ for $y$ in (175),

(178) $D(x)^{6} y D(x) g(x) z D(x) g(x)+D(x)^{7} y D(x) g(x) z g(x)=0, \quad x, y, z \in R$.

From (177) and (178),

$$
D(x)^{6} y D(x) g(x) z D(x) g(x)=0, \quad x, y, z \in R .
$$

From (179), we have

$$
D(x)^{6} y D(x) g(x) z D(x)^{6} y D(x) g(x)=0, \quad x, y, z \in R .
$$

Since $R$ is prime, (180) yields

$$
D(x)^{6} y D(x) g(x)=0, \quad x, y \in R .
$$

From (175) and (181),

$$
D(x)^{7} y g(x)=0, \quad x, y \in R .
$$

Replacing $y\left(f(x) D(x) f(x)+D(x) f(x)^{2}+D(x)^{2} g(x)\right) z$ for $y$ in (75),

$$
\begin{aligned}
& D(x)^{4} y\left(f(x) D(x) f(x)+D(x) f(x)^{2}+D(x)^{2} g(x)\right) z(f(x) D(x) f(x) \\
& \left.+D(x) f(x)^{2}+D(x)^{2} g(x)\right)+D(x)^{5} y\left(f(x) D(x) f(x)+D(x) f(x)^{2}\right. \\
& \left.+D(x)^{2} g(x)\right) z\left(f(x)^{2}+D(x) g(x)\right)+D(x)^{6} y(f(x) D(x) f(x) \\
(182) \quad & \left.+D(x) f(x)^{2}+D(x)^{2} g(x)\right) z g(x)=0, \quad x, y, z \in R .
\end{aligned}
$$

From (145), (174) and (182), we arrive at

$$
D(x)^{4} y\left(f(x) D(x) f(x)+D(x) f(x)^{2}+D(x)^{2} g(x)\right) z(f(x) D(x) f(x)
$$

From (183), we get

$$
\begin{aligned}
& D(x)^{4} y\left(f(x) D(x) f(x)+D(x) f(x)^{2}\right. \\
& \left.+D(x)^{2} g(x)\right) z D(x)^{4} y(f(x) D(x) f(x) \\
& \left.+D(x) f(x)^{2}+D(x)^{2} g(x)\right)=0, \quad x, y, z \in R .
\end{aligned}
$$


Since $R$ is prime, (184) gives

$$
D(x)^{4} y\left(f(x) D(x) f(x)+D(x) f(x)^{2}+D(x)^{2} g(x)\right)=0, \quad x, y \in R .
$$

From (75) and (185),

$$
D(x)^{5} y\left(f(x)^{2}+D(x) g(x)\right)+D(x)^{6} y g(x)=0, \quad x, y \in R .
$$

Replacing $y D(x) g(x) z$ for $y$ in (186),

$$
\begin{aligned}
& D(x)^{5} y D(x) g(x) z\left(f(x)^{2}+D(x) g(x)\right)+D(x)^{6} y D(x) g(x) z g(x) \\
= & 0, \quad x, y, z \in R .
\end{aligned}
$$

From (181) and (187), we obtain

$$
D(x)^{5} y D(x) g(x) z\left(f(x)^{2}+D(x) g(x)\right)=0, \quad x, y, z \in R .
$$

Replacing $y f(x)^{2} z$ for $y$ in (186),

(189) $D(x)^{5} y f(x)^{2} z\left(f(x)^{2}+D(x) g(x)\right)+D(x)^{6} y f(x)^{2} z g(x)=0, \quad x, y, z \in R$.

From (154) and (189),

$$
D(x)^{5} y f(x)^{2} z\left(f(x)^{2}+D(x) g(x)\right)=0, \quad x, y, z \in R .
$$

From (188) and (190),

$$
D(x)^{5} y\left(f(x)^{2}+D(x) g(x)\right) z\left(f(x)^{2}+D(x) g(x)\right)=0, \quad x, y, z \in R .
$$

From (191), we get

(192) $D(x)^{5} y\left(f(x)^{2}+D(x) g(x)\right) z D(x)^{5} y\left(f(x)^{2}+D(x) g(x)\right)=0, \quad x, y, z \in R$.

Since $R$ is prime, (192) gives

$$
D(x)^{5} y\left(f(x)^{2}+D(x) g(x)\right)=0, \quad x, y \in R .
$$

From (75), (185) and (193), we arrive at

$$
D(x)^{6} y g(x)=0, \quad x, y \in R .
$$

Left multiplication of (70) by $D(x)^{2}$ leads to

$$
\begin{aligned}
& D(x)^{3} y D(x)^{2} f(x)+D(x)^{2} f(x) D(y) D(x) f(x)+D(x)^{4} y D(x) f(x) \\
& +D(x)^{2} f(x) D(x) D(y) f(x)+D(x)^{3} f(x) D(y) f(x)+D(x)^{5} y f(x) \\
& +D(x)^{2} f(x) D(x)^{2} B(x, y)+D(x)^{3} f(x) D(x) B(x, y) \\
& +D(x)^{4} f(x) B(x, y)+D(x)^{6}[y, x]=0, \quad x, y \in R .
\end{aligned}
$$

From (55) and (195), we have

$$
\begin{aligned}
& D(x)^{3} y D(x)^{2} f(x)+D(x)^{2} f(x) D(y) D(x) f(x)+D(x)^{4} y D(x) f(x) \\
& +D(x)^{2} f(x) D(x) D(y) f(x)+D(x)^{5} y f(x)+D(x)^{2} f(x) D(x)^{2} B(x, y) \\
(196) \quad & +D(x)^{6}[y, x]=0, \quad x, y \in R .
\end{aligned}
$$


Right multiplication of (196) by $z g(x)$ leads to

$$
\begin{aligned}
& D(x)^{3} y D(x)^{2} f(x) z g(x)+D(x)^{2} f(x) D(y) D(x) f(x) z g(x) \\
& +D(x)^{4} y D(x) f(x) z g(x)+D(x)^{2} f(x) D(x) D(y) f(x) z g(x) \\
& +D(x)^{5} y f(x) z g(x)+D(x)^{2} f(x) D(x)^{2} B(x, y) z g(x) \\
& +D(x)^{6}[y, x] z g(x)=0, \quad x, y, z \in R .
\end{aligned}
$$

From (55), (194) and (197),

$$
\begin{aligned}
& D(x)^{3} y D(x)^{2} f(x) z g(x)+D(x)^{2} f(x) D(y) D(x) f(x) z g(x) \\
& +D(x)^{4} y D(x) f(x) z g(x)+D(x)^{2} f(x) D(x) D(y) f(x) z g(x) \\
(198) \quad & +D(x)^{5} y f(x) z g(x)+D(x)^{2} f(x) D(x)^{2} B(x, y) z g(x)=0, \quad x, y, z \in R .
\end{aligned}
$$

Left multiplication of (198) by $D(x)$ yields

$$
\begin{aligned}
& D(x)^{4} y D(x)^{2} f(x) z g(x)+D(x)^{3} f(x) D(y) D(x) f(x) z g(x) \\
&+D(x)^{5} y D(x) f(x) z g(x)+D(x)^{3} f(x) D(x) D(y) f(x) z g(x) \\
&(199) \quad+D(x)^{6} y f(x) z g(x)+D(x)^{3} f(x) D(x)^{2} B(x, y) z g(x)=0, \quad x, y, z \in R .
\end{aligned}
$$

From (55), (194) and (199),

$$
D(x)^{4} y D(x)^{2} f(x) z g(x)+D(x)^{5} y D(x) f(x) z g(x)=0, \quad x, y, z \in R .
$$

Replacing $y D(x)$ for $y$ in $(200)$,

$$
D(x)^{4} y D(x)^{3} f(x) z g(x)+D(x)^{5} y D(x)^{2} f(x) z g(x)=0, \quad x, y, z \in R .
$$

From (55) and (201), we get

$$
D(x)^{5} y D(x)^{2} f(x) z g(x)=0, \quad x, y, z \in R .
$$

Replacing $y D(x)^{2} f(x) w$ for $y$ in (200),

$$
\begin{aligned}
& D(x)^{4} y D(x)^{2} f(x) w D(x)^{2} f(x) z g(x) \\
& +D(x)^{5} y D(x)^{2} f(x) w D(x) f(x) z g(x)=0, \quad w, x, y, z \in R .
\end{aligned}
$$

From (202) and (203),

$$
D(x)^{4} y D(x)^{2} f(x) w D(x)^{2} f(x) z g(x)=0, \quad w, x, y, z \in R .
$$

From (204),

(205) $D(x)^{4} y D(x)^{2} f(x) z g(x) w D(x)^{4} y D(x)^{2} f(x) z g(x)=0, \quad w, x, y, z \in R$.

Since $R$ is prime, (205) yields

$$
D(x)^{4} y D(x)^{2} f(x) z g(x)=0, \quad x, y, z \in R .
$$

From (200) and (206),

$$
D(x)^{5} y D(x) f(x) z g(x)=0, \quad x, y, z \in R .
$$


Left multiplication of (198) by $D(x)^{5} w$ leads to

$$
\begin{aligned}
& D(x)^{5} w D(x)^{3} y D(x)^{2} f(x) z g(x) \\
& +D(x)^{5} w D(x)^{4} y D(x) f(x) z g(x) \\
& +D(x)^{5} w D(x)^{2} f(x) D(y) D(x) f(x) z g(x) \\
& +D(x)^{5} w D(x)^{2} f(x) D(x) D(y) f(x) z g(x)+D(x)^{5} w D(x)^{5} y f(x) z g(x) \\
(208) \quad & +D(x)^{5} w D(x)^{2} f(x) D(x)^{2} B(x, y) z g(x)=0, \quad w, x, y, z \in R .
\end{aligned}
$$

From (207) and (208),

$$
D(x)^{5} w D(x)^{5} y f(x) z g(x)=0, \quad w, x, y, z \in R .
$$

From (209), we have

$$
D(x)^{5} y f(x) z g(x) w D(x)^{5} y f(x) z g(x)=0, \quad w, x, y, z \in R .
$$

Since $R$ is prime, (210) gives

$$
D(x)^{5} y f(x) z g(x)=0, \quad x, y, z \in R .
$$

A simple calculation shows that (211) yields

$$
D(x)^{5} y g(x)=0, \quad x, y \in R .
$$

From (212), by Lemma 3.3 we have

$$
D(x)=0, \quad x \in R .
$$

\section{Applications in Banach algebra theory}

The following theorem is proved by the same arguments as in the proof of J. Vukman's theorem [15], but it generalizes his result.

Theorem 4.1. Let $A$ be a Banach algebra. Suppose there exists a continuous linear Jordan derivation $D: A \longrightarrow A$ such that

$$
D(x)^{3}[D(x), x] \in \operatorname{rad}(A)
$$

for all $x \in A$. Then we have $D(A) \subseteq \operatorname{rad}(A)$.

Proof. It suffices to prove the case that $A$ is noncommutative. By the result of B. E. Johnson and A. M. Sinclair [5] any linear derivation on a semisimple Banach algebra is continuous. Sinclair [11] has proved that every continuous linear Jordan derivation on a Banach algebra leaves the primitive ideals of $A$ invariant. Hence for any primitive ideal $P \subseteq A$ one can introduce a derivation $D_{P}: A / P \longrightarrow A / P$, where $A / P$ is a prime and factor Banach algebra, by $D_{P}(\hat{x})=D(x)+P, \hat{x}=x+P$. By the assumption that $D(x)^{3}[D(x), x] \in$ $\operatorname{rad}(A), x \in A$, we obtain $\left(D_{P}(\hat{x})\right)^{3}\left[D_{P}(\hat{x}), \hat{x}\right]=0, \hat{x} \in A / P$, since all the assumptions of Theorem 3.4 are fulfilled. Let the factor prime Banach algebra $A / P$ be noncommutative. Then we have $D_{P}(\hat{x})=0, \hat{x} \in A / P$. Thus we obtain $D(x) \in P$ for all $x \in A$ and all primitive ideals of $A$. Hence $D(A) \subseteq$ $\operatorname{rad}(A)$. And we consider the case that $A / P$ is commutative. Then since $A / P$ is a commutative Banach semisimple Banach algebra, from the result of B. E. 
Johnson and A. M. Sinclair [5], it follows that $D_{P}(\hat{x})=0, \hat{x} \in A / P$. And so, $D(x) \in P$ for all $x \in A$ and all primitive ideals of $A$. Hence $D(A) \subseteq \operatorname{rad}(A)$. Therefore in any case we obtain $D(A) \subseteq \operatorname{rad}(A)$.

Theorem 4.2. Let $A$ be a semisimple Banach algebra. Suppose there exists a linear Jordan derivation $D: A \longrightarrow A$ such that

$$
D(x)^{3}[D(x), x]=0
$$

for all $x \in A$. Then we have $D=0$.

Proof. It suffices to prove the case that $A$ is noncommutative. According to the result of B. E. Johnson and A. M. Sinclair [5] every linear derivation on a semisimple Banach algebra is continuous. A. M. Sinclair [11] has proved that any continuous linear derivation on a Banach algebra leaves the primitive ideals of $A$ invariant. Hence for any primitive ideal $P \subseteq A$ one can introduce a derivation $D_{P}: A / P \longrightarrow A / P$, where $A / P$ is a prime and factor Banach algebra, by $D_{P}(\hat{x})=D(x)+P, \hat{x}=x+P$. From the given assumptions $D(x)^{3}[D(x), x]=0, x \in A$, it follows that $\left(D_{P}(\hat{x})\right)^{3}\left[D_{P}(\hat{x}), \hat{x}\right]=0, \hat{x} \in A / P$, since all the assumptions of Theorem 3.4 are fulfilled. The factor algebra $A / P$ is noncommutative, by Theorem 3.4 we have $D_{P}(\hat{x})=0, \hat{x} \in A / P$. Hence we get $D(A) \subseteq P$ for all primitive ideals $P$ of $A$. Thus $D(A) \subseteq \operatorname{rad}(A)$. But since $A$ is semisimple, $D=0$.

As a special case of Theorem 4.2 we get the following result which characterizes commutative semisimple Banach algebras.

Corollary 4.3. Let $A$ be a semisimple Banach algebra. Suppose

$$
[x, y]^{3}[[x, y], x]=0
$$

for all $x, y \in A$. In this case, $A$ is commutative.

\section{References}

[1] F. F. Bonsall and J. Duncan, Complete Normed Algebras, Berlin-Heidelberg-New York, 1973.

[2] M. Brešar, Derivations of noncommutative Banach algebras. II, Arch. Math. 63 (1994), no. 1, 56-59.

[3] _ Jordan derivations on semiprime rings, Proc. Amer. Math. Soc. 104 (1988), no. 4, 1003-1006.

[4] L. O. Chung and J. Luh, Semiprime rings with nilpotent derivatives, Canad. Math. Bull. 24 (1981), no. 4, 415-421.

[5] B. E. Johnson and A. M. Sinclair, Continuity of derivations and a problem of Kaplansky, Amer. J. Math. 90 (1968), 1067-1073.

[6] B. D. Kim, On the derivations of semiprime rings and noncommutative Banach algebras, Acta Math. Sin. (Engl. Ser.) 16 (2000), no. 1, 21-28.

[7] _ Derivations of semiprime rings and noncommutative Banach algebras, Commun. Korean Math. Soc. 17 (2002), no. 4, 607-618.

[8] J Jordan derivations of semiprime rings and noncommutative Banach algebras. I, J. Korea Soc. Math. Educ. Ser. B. Pure Appl. Math. 15 (2008), no. 2, 179-201. 
[9] J Jordan derivations of semiprime rings and noncommutative Banach algebras. II, J. Korea Soc. Math. Educ. Ser. B. Pure Appl. Math. 15 (2008), no. 3, 259-296.

[10] K. H. Park and B. D. Kim, On continuous linear Jordan derivations of Banach algebras, J. Korea Soc. Math. Educ. Ser. B. Pure Appl. Math. 16 (2009), no. 2, 227-241.

[11] A. M. Sinclair, Jordan homomorphisms and derivations on semisimple Banach algebras, Proc. Amer. Math. Soc. 24 (1970), 209-214.

[12] I. M. Singer and J. Wermer, Derivations on commutative normed algebras, Math. Ann. 129 (1955), 260-264.

[13] M. P. Thomas, The image of a derivation is contained in the radical, Ann. of Math. 128 (1988), no. 3, 435-460.

[14] J. Vukman, A result concerning derivations in noncommutative Banach algebras, Glas. Mat. Ser. III 26(46) (1991), no. 1-2, 83-88.

[15] - On derivations in prime rings and Banach algebras, Proc. Amer. Math. Soc. 116 (1992), no. 4, 877-884.

Department of Mathematics

Gangneung-Wonju National University

Gangneung 210-702, Korea

E-mail address: bdkim@gwnu.ac.kr 